\title{
COMO as MolÉCULAS Sublimam...
}

\section{CARLOS LIMA}

equilíbrio de sublimação consiste no equilíbrio termodinâmico estabelecido, a uma determinada temperatura, entre as fases sólida e gasosa de uma substância pura, estando já bem compreendido pela comunidade científica. No entanto, a um nível mecanístico e molecular, este fenómeno é mais complexo do que parece. Neste artigo, o equilíbrio de sublimação de um sólido molecular cristalino é abordado numa perspetiva cinética e dissecado nos seus dois passos elementares: a sublimação (quando as moléculas adquirem energia suficiente e escapam do sólido) e a condensação (quando as moléculas gasosas colidem com a superfície do sólido e são incorporadas na malha cristalina).

\section{INTRODUÇÃO}

O trabalho de um químico pode dividir-se em dois objetivos principais: usar as moléculas para compreender as leis da natureza e usar as leis da natureza para compreender as moléculas. Este artigo foca-se no primeiro objetivo. As moléculas utilizadas consistem num conjunto de fenilbenzenos e fenilnaftalenos (Figura 1), escolhidas com o intuito de explorar, ao nível molecular, a cinética de sublimação.

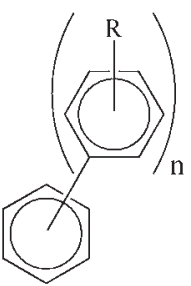

(A)

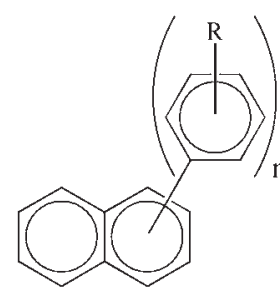

(B)
Figura 1 - Representação esquemática dos fenilbenzenos (A) e fenilnaftalenos (B) estudados; $\mathrm{R}=$ fenil ou $\mathrm{H}, \mathrm{n}=1-6$

De um ponto de vista mecanístico, o processo de sublimação consiste em dois passos elementares: 1) sublimação - escape de moléculas do sólido para a fase gasosa; 2) condensação - impacto de moléculas na superfície do sólido e sua incorporação na maIha cristalina. Cada um destes passos ocorre incessantemente e a uma velocidade que depende de muitos fatores, tais como temperatura, forças intermoleculares, simetria e flexibilidade moleculares. Quando as velocidades destes dois passos se igualam, atinge-

\footnotetext{
* Centro de Investigação em Química, Departamento de Química e Bioquímica, Faculdade de Ciências da Universidade do Porto

E-mail: carlos.chemistry@gmail.com
}

-se o equilíbrio entre as fases sólida e gasosa, traduzido macroscopicamente por uma pressão de vapor de saturação. É o balanço destes dois passos elementares que define a posição de equilíbrio de sublimação e estabelece qual a pressão de vapor de um sólido a uma determinada temperatura.

\section{NO LABORATÓRIO}

As pressões de vapor em função da temperatura foram medidas para os compostos estudados, permitindo derivar as entalpias, $\Delta_{\text {sub }} H_{m}{ }^{0}$, entropias, $\Delta_{\text {sub }} S_{m}{ }^{0}$, e energias de Gibbs, $\Delta_{\text {sub }} G_{m}{ }^{0}$, molares de sublimação padrão a uma temperatura de referência (normalmente 298,15 K). Enquanto as entalpias nos dizem quão forte é a coesão entre as moléculas na fase sólida, estando diretamente relacionadas com as forças intermoleculares, as entropias traduzem o grau de liberdade translacional, rotacional e vibracional que as moléculas obtêm ao transitar de um meio cristalino obstruído e ordenado para um meio gasoso desobstruído e caótico.

\section{DEFINIR O PROBLEMA}

Define-se agora claramente o objeto de estudo deste trabalho e o tipo de sistema considerado (Figura 2):

- Objeto de estudo - cinética de sublimação e seus dois passos elementares: sublimação e condensação.

- Tipo de sistema - sólido molecular cristalino, cuja fase gasosa é constituída por moléculas isoladas e estáveis nas condições consideradas.

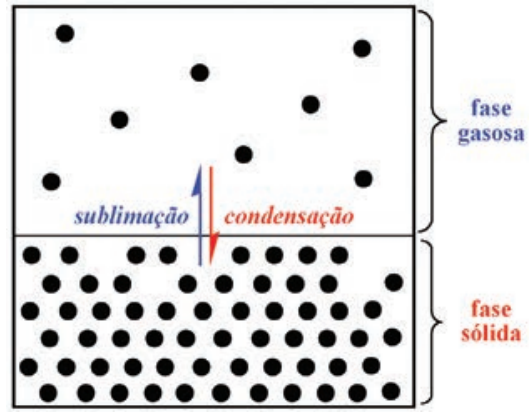

Figura 2 - Esquema do objeto de estudo deste trabalho e sistema considerado

SUBLIMAÇÃO - QUANDO ELAS ESCAPAM

No estado sólido, as moléculas estão confinadas a um potencial intermolecular que as mantém coesas e ordenadas. Este potencial resulta das forças intermoleculares que atuam entre moléculas vizinhas e que dependem da molécula em questão. Para sublimar, uma molécula necessita de superar o potencial intermolecular e escapar livremente para a fase gasosa. Para isso é preciso energia e, nas condições em que o sólido é a fase mais estável, a energia média por molécula não é suficiente para que elas se separem (se assim fosse, a fase mais estável seria o líquido ou o gás). A molécula precisa então de pedir emprestado um excesso momentâneo de energia às suas vizinhas. Estas oscilações térmicas permitem que a molécula adquira num dado momento energia cinética suficiente para vencer a barreira das forças intermoleculares e sublimar. No entanto, as moléculas levam consigo o excesso de energia emprestado, deixando para trás um sólido arrefecido, o que corrobora a 
natureza endotérmica da sublimação. É lógico admitir que as moléculas que efetivamente sublimam são aquelas que se encontram à superfície do sólido (as outras estão demasiado obstruídas para conseguirem escapar) e que quanto maior for a área dessa superfície, maior será a velocidade de sublimação. A lei cinética de velocidades para o processo elementar da sublimação toma então a forma:

$\mathrm{v}_{\text {sub }}=\mathrm{k}_{\text {sub }} \cdot \mathrm{S}$

onde $v_{\text {sub }}$ é a velocidade de sublimação (pode ser expressa em moléculas/s), $k_{\text {sub }}$ a constante de velocidade de sublimação e $S$ a área de superfície do sólido; segundo a equação de Arrhenius:

$\mathrm{k}_{\text {sub }}=\mathrm{A}_{\text {sub }} \cdot \exp \left(-\mathrm{E}_{\mathrm{a}, \text { sub }} / \mathrm{RT}\right)$

sendo $A_{\text {sub }} \circ$ fator pré-exponencial, $E_{\text {a,sub }}$ a energia de ativação, $R$ a constante dos gases e $T$ a temperatura. $E_{\mathrm{a} \text {,sub }}$ corresponde à barreira energética que as moléculas têm que ultrapassar para sublimar e relaciona-se diretamente com a energia coesiva do sólido, que por sua vez está intimamente relacionada com $\Delta_{\text {sub }} H_{m}{ }^{0}$. No sólido, as moléculas ocupam uma posição bem definida e oscilam em torno desta com uma amplitude que é proporcional ao seu conteúdo energético. Normalmente, estas oscilações são pequenas e insuficientes para que a molécula se afaste consideravelmente da sua posição de equilíbrio. Quando, aleatoriamente, a molécula adquire um excesso de energia, as oscilações tornam-se mais vigorosas e a molécula afasta-se da sua posição de equilíbrio até distâncias a que pode já não estar sujeita à prisão do potencial intermolecular, sublimando. Como se pode constatar pela equação (2), admitindo que $E_{a \text {,sub }}>0$, o aumento de temperatura aumenta $k_{\text {sub }}$, uma vez que a energia média das moléculas é maior e portanto mais moléculas por unidade de tempo adquirem energia suficiente para vencer $E_{\mathrm{a}, \mathrm{sub}}$. Para que estes conceitos se tornem visualizáveis, podemos definir o potencial intermolecular como a entalpia de interação intermolecular, $H_{\text {inter }}$ $\left(H_{\text {inter }} \approx \Delta_{\text {sub }} H_{\mathrm{m}}{ }^{0}\right)$, e focarmo-nos numa molécula na superfície de um sólido esférico, como ilustrado na Figura 3. A distância de equilíbrio dessa molécula ao centro da esfera, C, é traduzida por
$d_{C}\left(\right.$ eq) e $d_{C}(I)$ traduz a distância limite acima da qual a molécula já não experiencia o potencial intermolecular e sublima livremente. Se considerarmos comportamento de gás ideal, vem que $H_{\text {inter }}=0$ na fase gasosa.

Um outro fator que também influencia $v_{\text {sub }}$ é a pressão atmosférica. As moléculas gasosas embatem com muita frequência na superfície do sólido. $O$ efeito da pressão pode ser visto como um incremento adicional em $E_{\mathrm{a}, \text { sub }}$, devido às repulsões locais que as moléculas à superfície experimentam com um número médio de moléculas gasosas imediatamente acima. À medida que a pressão aumenta as moléculas do sólido sublimam mais devagar, visto terem mais obstáculos no caminho (Figura 4).

Na Figura 5 apresentam-se os resultados de $\Delta_{\text {sub }} H_{m}{ }^{0}$ para três fenilnaftalenos estudados.

Com o aumento do tamanho da molécula aumenta também $\Delta_{\text {sub }} H_{m}{ }^{0}$. Isto significa que as forças coesivas se tornam mais intensas, sendo necessário mais energia para separar as moléculas e enviá-las para a fase gasosa. Assim, para a mesma temperatura, as moléculas do composto maior

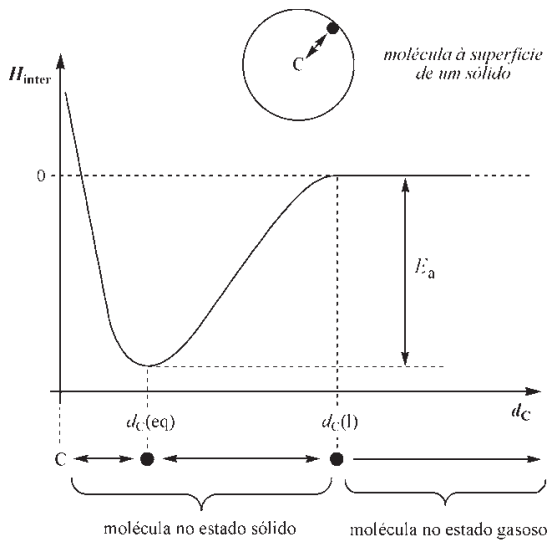

Figura 3 - Esquema da curva $H_{\text {inter }}=f\left(d_{c}\right)$ para uma molécula à superfície de um sólido esférico

precisam de mais tempo para se separar, apresentando menor velocidade de sublimação.

\section{CONDENSAÇÃO - QUANDO BATEM E FICAM}

O passo elementar da condensação é ligeiramente mais complexo que o da sublimação. Neste passo as moléculas devem incorporar-se corretamente numa malha cristalina ordenada e regular. $\mathrm{O}$ primeiro fator a considerar é a frequência de embate na superfície sólida, bem como a área dessa superfície. Quanto mais frequentes os embates (ou quanto maior a pressão) e quanto maior área, mais moléculas condensarão por unidade de tempo. A lei cinética de condensação é então dada por:

$\mathrm{v}_{\text {cond }}=\mathrm{k}_{\text {cond }} \cdot \mathrm{p} \cdot \mathrm{S}$

onde $p$ é a pressão de vapor do composto, não necessariamente a pressão de equilíbrio. A dependência de $k_{\text {cond }}$ com $T$ pode ser expressa como:

$\mathrm{k}_{\text {cond }}=\mathrm{A}_{\text {cond }} \cdot \exp \left(-\mathrm{E}_{\mathrm{a}, \text { cond }} / \mathrm{RT}\right)$

A parte mais subtil vem a seguir. Será $E_{\mathrm{a}, \mathrm{cond}}$ maior, igual ou menor que zero? Que fatores influenciam $k_{\text {cond }}$ ? Para responder a estas questões é necessário descer novamente ao micromundo das moléculas. Imaginemos então que somos encolhidos à escala molecular e vamos dar um passeio pela superfície de um sólido em equi-

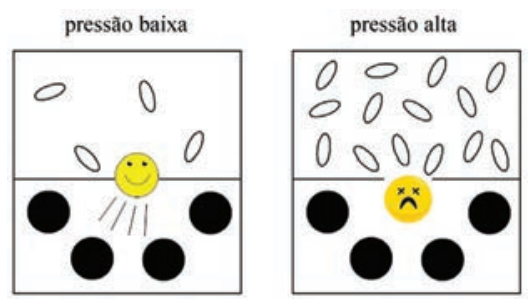

Figura 4-Imagem ilustrativa do efeito da pressão atmosférica na velocidade de sublimação

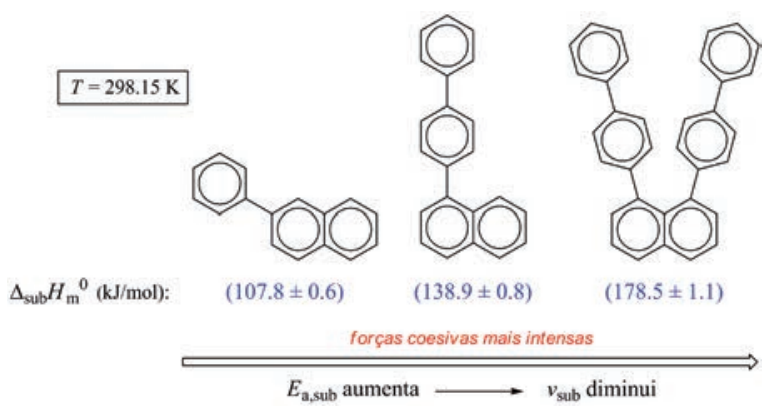

Figura $5-\Delta_{\text {sub }} H_{m}{ }^{0}$ para alguns fenilnaftalenos e sua relação com a cinética de sublimação 
líbrio com o seu vapor. Antes de mais temos de nos proteger das imensas moléculas que incessantemente sublimam e daquelas que passeiam na fase gasosa a velocidades aterradoras, assim como devemos tomar especial cuidado às irregularidades do chão que pisamos, pois uma superfície cristalina assemelha-se mais a um terreno montanhoso do que a uma estrada bem alcatroada. E se queremos observar algo que se preze convém abrandar o tempo à escala dos nanosegundos.

No momento do impacto com a superfície, as moléculas possuem uma certa quantidade de energia cinética. Parte dela é dissipada quando as moléculas se aproximam da posição de equilíbrio que ocupam no cristal e começam a sentir o efeito atrativo das forças intermoleculares. No entanto, se a energia cinética da molécula for demasiado alta relativamente à velocidade com que essa energia é dissipada, a ação do potencial intermolecular não vai conseguir imobilizar eficazmente a molécula incidente e provavelmente ela será refletida de volta para a fase gasosa em vez de condensar. Segundo esta lógica, um aumento da temperatura contribui para uma diminuição de $k_{\text {cond }}$, uma vez que aumenta a energia cinética média das moléculas e logo a probabilidade de serem refletidas. De acordo com a equação (4), uma diminuição de $k_{\text {cond }}$ com o aumento de temperatura implica uma $E_{\mathrm{a} \text {,cond }}$ negativa, algo que não é muito comum observar-se em reações químicas.

Podemos imaginar o processo de condensação como um arremesso contínuo de peças ao seu mosaico correspondente e ver quantas lá encaixam por unidade de tempo, ou melhor, do total de peças arremessadas, qual a fração de peças que bate e fica. A esta fração chama-se, na gíria científica, coeficiente de condensação. Todas as peças têm a mesma forma e uma posição bem definida no mosaico. É de reparar, como ilustrado na Figura 6 , que formas mais simétricas possuem mais orientações compatíveis com o espaço que a peça ocupa no mosaico. Enquanto que um quadrado apresenta oito orientações espaciais distintas (resultantes de 4 rotações de

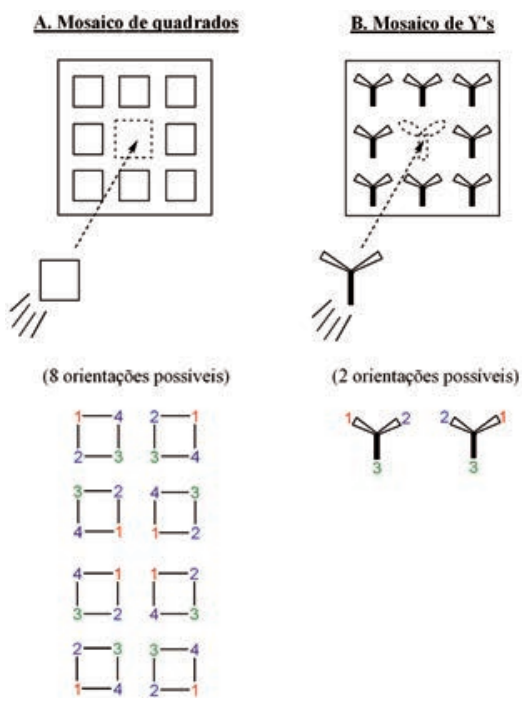

Figura 6 - Num arremesso aleatório, a maior simetria da peça quadrada leva a uma maior probabilidade de embate correto e incorporação no respetivo mosaico. Num embate mal orientado, a peça não se consegue incorporar no padrão do mosaico e é refletida

$90^{\circ}$ e 4 eixos de reflexão - horizontal, vertical e 2 diagonais) os Y's apresentam apenas duas. Assim, construir um mosaico de Y's exige mais tempo e esforço do que construir um mosaico igualmente grande de quadrados (é necessário um esforço extra para orientar os Y's corretamente).

A transposição deste raciocínio para o mundo molecular é imediata. Um sólido cristalino tem uma estrutura bem ordenada e cada molécula ocupa uma posição de equilíbrio bem definida. Cada nova molécula incorporada tem de adquirir uma orientação correta relativamente às suas vizinhas. Sendo assim, a orientação relativa das moléculas gasosas aquando do impacto com a superfície do sólido afeta a probabilidade de condensação. Se a molécula choca com a orientação correta, a probabilidade de condensar será apenas influenciada pela energia cinética que possui nesse momento. No entanto, se a molécula choca com uma orientação muito distinta daquela que deveria adotar na malha cristalina, vai sentir um potencial intermolecular mais fraco e demorar mais tempo a atingir a posição de equilíbrio, aumentando a probabilidade de ser refletida.

A Figura 7 ilustra um caso geral onde a molécula bate com a orientação correta, [a], e incorreta, [b].

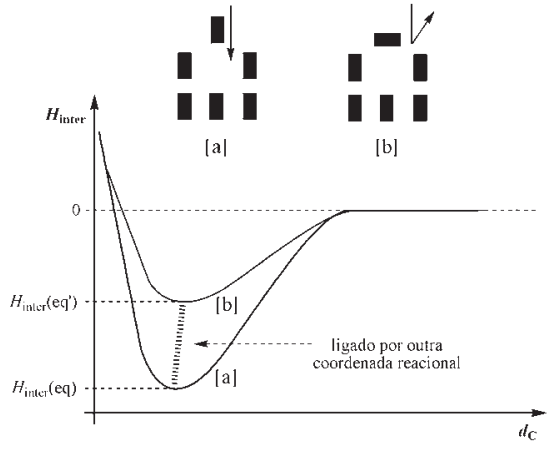

Figura 7 - Curva $H_{\text {inter }}=f\left(d_{c}\right)$ para uma molécula que bate no sólido cristalino com uma orientação correta, [a], e incorreta, [b]

Em [a] a molécula atinge facilmente o mínimo absoluto em $H_{\text {inter }}$ e tem maior probabilidade de condensar. Em [b] a molécula pode conseguir atingir um mínimo relativo em $H_{\text {inter }}$ e então relaxar para o mínimo absoluto por intermédio de uma outra coordenada reacional (por exemplo, rodando em torno de si própria). No entanto, antes que a molécula tenha tempo para relaxar o excesso de energia cinética ainda não dissipado, pode jogar a sua cartada e enviar a molécula de volta para o gás. Desta forma, moléculas mais simétricas apresentam maior probabilidade de condensar porque a maior simetria aumenta o número de orientações corretas da molécula relativamente à malha cristalina. Este número é igual ao número de simetria externa de uma molécula, $\sigma_{\text {sim }}$, definido como o número de orientações únicas de uma molécula rígida que apenas permutam átomos idênticos. Maior $\sigma_{\text {sim }}$ aumenta a probabilidade de condensação, o que pode ser traduzido matematicamente pela discriminação deste número na equação (4), resultando em:

$\mathrm{k}_{\text {cond }}=\sigma_{\text {sim }} \cdot \mathrm{A}_{\text {cond }}^{\prime} \cdot \exp \left(-\mathrm{E}_{\mathrm{a} \text {, cond }} / \mathrm{RT}\right)$

onde foi considerado que

$A_{\text {cond }}=\sigma_{\text {sim }} \cdot A_{\text {cond }}^{\prime}$

Um outro fator que pode influenciar $k_{\text {cond }}$ é a flexibilidade molecular, que se traduz na possibilidade da molécula poder alterar a sua geometria adquirindo outras conformações estáveis ou deformando-se significativamente devido a rotações internas de grupos flexíveis. Este facto pode levar a uma dificuldade acrescida em cristalizar, 
uma vez que na fase gasosa a molécula vai adotando ao longo do tempo algumas geometrias incompatíveis com a fase cristalina (Figura 8). Uma vez que a malha cristalina é altamente seletiva, estas geometrias apresentam menor probabilidade de efetivamente condensar, diminuindo $k_{\text {cond }}$.

As contribuições da simetria e flexibilidade refletem-se termodinamicamente em $\Delta_{\text {sub }} S_{m}{ }^{0}$. Mais simetria está associada a um aumento da entropia do sólido, logo menor $\Delta_{\text {sub }} S_{m}{ }^{0}$, porque existem mais formas equivalentes de construir a malha cristalina. Mais flexibilidade está associada a mais liberdade conformacional, que se faz sentir principalmente na fase gasosa (no cristal o potencial intermolecular restringe a molécula a uma geometria), levando a um aumento da entropia do gás e, consequentemente, de $\Delta_{\text {sub }} S_{m}{ }^{0}$. $\mathrm{Na}$ Figura 9 ilustra-se este raciocínio utilizando dois fenilbenzenos estudados.

\section{POR FIM O EQUILÍBRIO}

O equilíbrio é o resultado harmonioso deste incessante e competitivo reboliço molecular; enquanto a sublimação desfaz o sólido, a condensação reconstrói-o. A situação de equilíbrio, traduzida por uma pressão de vapor de saturação, corresponde às condições a que as duas velocidades se igualam, $v_{\text {sub }}=v_{\text {cond }}$ para $p=p_{\text {eq }}(\mathrm{Fi}-$ gura 10). Rearranjando as equações anteriormente apresentadas vem:

$p_{\text {eq }}=\frac{k_{\text {sub }} \cdot S}{k_{\text {cond }} \cdot S}=\frac{A_{\text {sub }} \cdot \exp \left(-E_{a, \text { sub }} / R T\right)}{\sigma_{\text {sim }} \cdot A_{\text {cond }}^{\prime} \cdot \exp \left(-E_{a, \text { cond }} / R T\right)}$

donde se constata facilmente que mais simetria e maior $\Delta_{\text {sub }} H_{\mathrm{m}}{ }^{0}$ (proporcional a $E_{\mathrm{a} \text {,sub }}$ ) diminuem a pressão de vapor de uma substância, tornando-a por isso menos volátil.

Neste artigo, o casamento entre cinética e termodinâmica torna-se claro e é fácil ver como os dois se relacionam e podem ser compreendidos usando a imaginação para viajar ao pequeno mundo das moléculas.
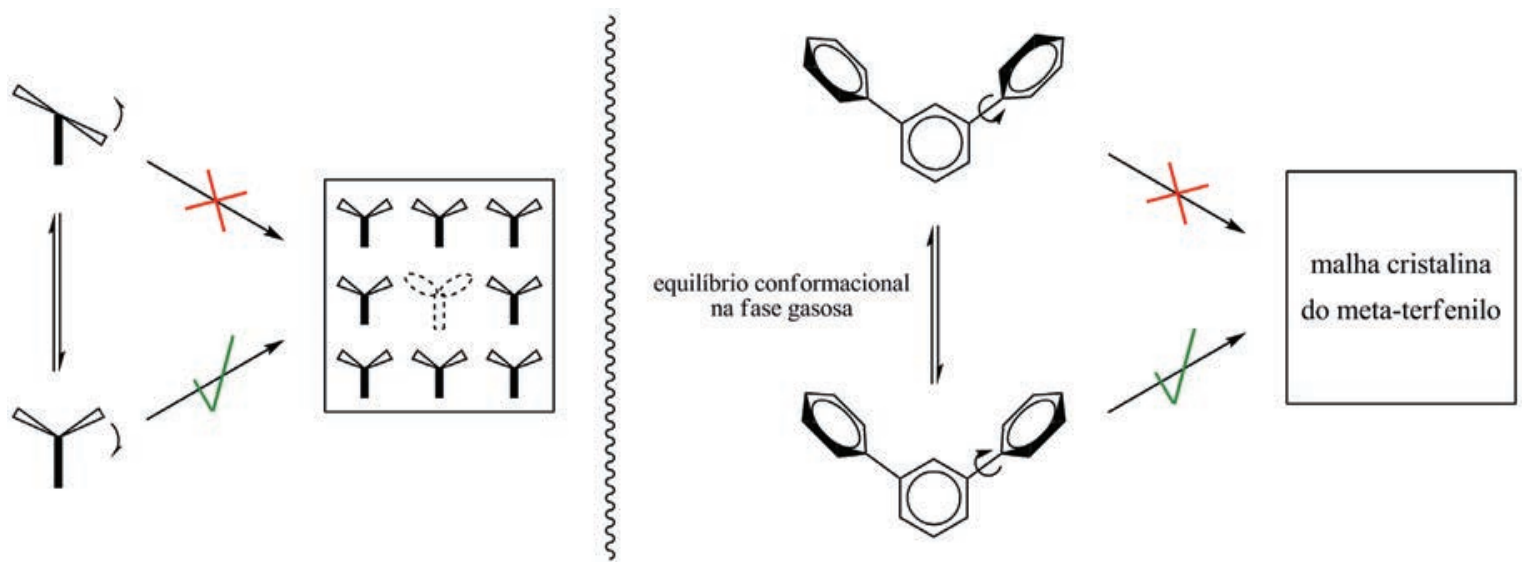

Figura 8 - A possibilidade de a molécula adotar outras conformações na fase gasosa pode diminuir a probabilidade de condensação, uma vez que apenas a conformação correta cristaliza

\section{Geometrias na fase cristalina}

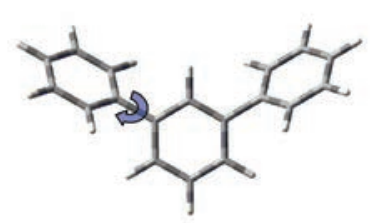

Baixa simetria: $\sigma_{\text {sim }}=1$

Flexibilidade: Rotação interna dos grupos fenil pouco impedida

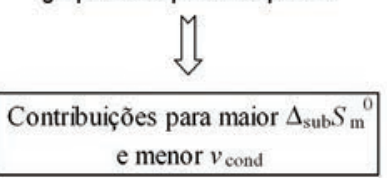

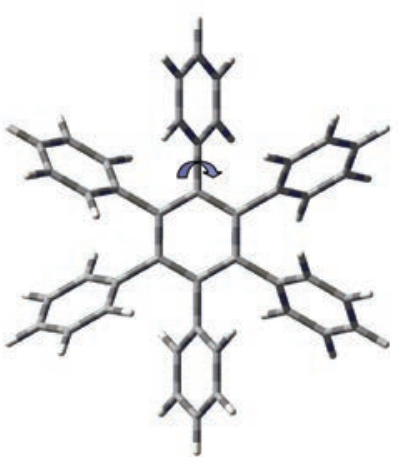

Elevada simetria: $\sigma_{\operatorname{sim}}=12$ Flexibilidade: Rotação interna dos grupos fenil muito impedida
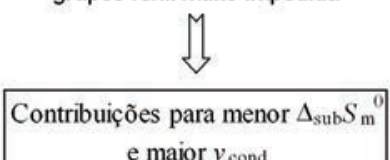

e maior $v_{\text {cond }}$

Figura 9 - Exemplos da influência da simetria e flexibilidade moleculares em dois fenilbenzenos

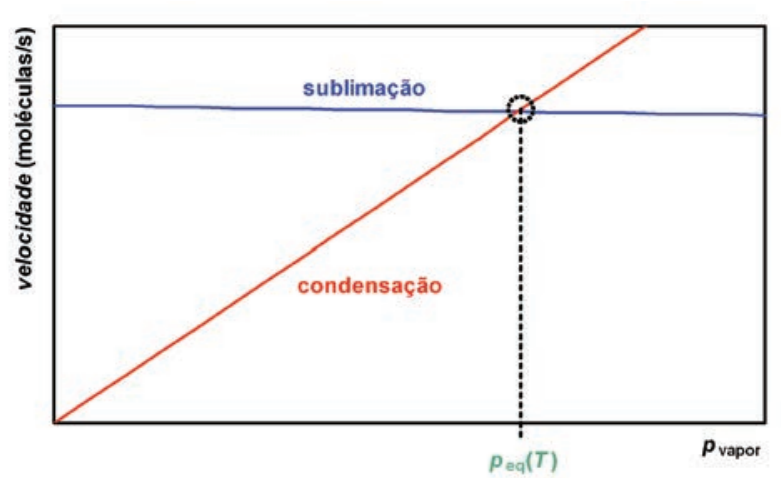

Figura 10 - Velocidades de sublimação e condensação em função da pressão de vapor, a temperatura constante; o equilíbrio atinge-se quando estas velocidades se igualam 